\title{
No-holes transpectoral tenodesis technique vs tenotomy of the long head of the biceps brachii
}

\author{
Enrico Gervasi \\ Enrico Sebastiani \\ Enrico Cautero
}

Department of Orthopaedics and Traumatology, Ospedale Civile di Latisana (UD), Italy

\author{
Corresponding author: \\ Enrico Sebastiani \\ Department of Orthopaedics and Traumatology, \\ Ospedale Civile di Latisana \\ Via Sabbionera 45 \\ 33053 Latisana (UD), Italy \\ E-mail: eseba@hotmail.it
}

\section{Summary}

Background: There is no univocal consensus regarding Long Head of the Biceps (LHB) best treatment between tenotomy and tenodesis. There is no consensus regarding the best location to perform the tenodesis. The LHB tenodesis performed by the proximal tendon excision as first step can miss the proper tension to the muscle belly. Fixations proximal to the pectoralis major can lead to groove pain. This study aims to test the efficacy of a new LHB tenodesis technique by comparing its results with the tenotomy.

Methods: We retrospectively evaluated patients who underwent surgery between May 2014 and May 2015. The mean follow up was 14.7 months. Sixteen patients underwent mini-open tenodesis to the Pectoralis Major tendon by the use of a resorbable suture (TD group); sixteen underwent tenotomy (TT group). The mean age of the TD group was 54 years; the mean age of the TT group was 56 years. We evaluated pain, subjective perception of the patient of possible aesthetic and strength differences between the two biceps, "Popeye sign", and tests to stimulate the LHB. We administered three evaluation questionnaires: the ASES score, the SPADI score, and the SST.

Results: 32 consecutive patients were evaluated. The clinical scores did not record statistically significant differences: the mean ASES score was 92.9 (TD) and 90.8 (TT); the mean SPADI score was 92.5 (TD), and 89.7 (TT); the mean SST was 8.9 (TD), and 8.4 (TT). Compared to the TD group, in the TT group we registered with greater frequency the "Popeye sign" with a P value $<0.05(9$ cases vs 1), and spasms in the biceps muscle belly (5 cases vs 1 ). All other signs or symptoms evaluated were more frequent in the TT group, except the strength difference perceived by the patient ( 3 patients in the TT group, and 2 in the TD group). No complications were recorded.

Conclusions: This new Long Head of the Biceps (LHB) tenodesis technique is valuable and reliable, and provided better results than tenotomy.

Level of evidence: IV.

KEY WORDS: long head of biceps, tenodesis.

\section{Introduction}

The tendon of the Long Head of the Biceps brachii (LHB) is often involved in shoulder pathologies; still, there is no univocal consensus regarding its best treatment between tenotomy and tenodesis ${ }^{1,2}$. Tenotomy is a simple and safe procedure that significantly reduces pain and allows a faster rehabilitation than tenodesis. Tenodesis allows maintaining the muscle length of the LHB, thus avoiding the aesthetic imperfection represented by the "Popeye Sign", reducing the risk of spasms, and improving biceps function ${ }^{3}$. There is an ongoing debate about the type of fixation to be used, and optimal tenodesis location: into the bicipital groove, suprapectoral or subpectoral. Tendon fixation to the groove can cause pain persistence, since this anatomical region is rich of vascularization and nociceptors ${ }^{4}$; therefore, several Authors prefer performing a subpectoral tenodesis with different fixation methods: trans-bone holes, suture anchors, interference screws, suspensory fixations ${ }^{5}$. Among the complications associated with these forms of fixation to the humeral diaphysis, we note iatrogenic nerve lesions and fractures caused by weakening of the humeral cortex ${ }^{6,7}$. In prosthetic surgery, we generally perform LHB tenodesis to the pectoralis major tendon using resorbable suture; this technique is rapid and cost-effective, it does not weaken the diaphysis by requiring bone holes, nor put at risk nerves on the posterior side. On these bases, we started to perform it during arthroscopic surgeries by using an anterior mini-open access. In this paper, we outline the results of this technique by comparing it with the tenotomies performed during the same period. Our hypothesis is that this new tenodesis tech- 
nique provides better results than tenotomy in term of popeye deformity, muscle belly spasm and pain.

\section{Matherials and methods}

We retrospectively recruited patients who underwent a LHB mini-open tenotomy or tenodesis to the Pectoralis Major tendon at the Ospedale Civile of Latisana between May 2014 and May 2015. Exclusion criteria include: patients older than 75 ; neurological lesions; cuff lesions involving the subscapularis; lesions of several cuff tendons or lesions whose repair required more than two anchors; irreparable cuff lesions; functional repairs and repairs associated with instability; patients who already underwent LHB tenodesis; spontaneous LHB rupture; combined Mumford procedures; previous fractures; suspected or documented rheumatic pathology; significant chondral lesions; other surgeries to the shoulder combined with tenodesis.

Each patient was asked about pain in the anterior region of the shoulder, episodes of spasms, strength difference in elbow flexion or supination, perceived aesthetic difference between the two biceps and if it would represent an issue. We performed the Yergason test and the Speed's test; we evaluated the eventual pain during pressure in the bicipital groove or the presence of the "Popeye sign". We administered three evaluation questionnaires: the American Shoulder and Elbow Surgeons Standardized Shoulder Assessment Form (ASES) ${ }^{8}$, Shoulder Pain and Disability Index (SPADI) ${ }^{9}$, and the Simple Shoulder Test $(\mathrm{SST})^{9}$, translated and validated in Italian.
This research has been conduct ethically according to international standards ${ }^{10}$.

We identify the presence of a "Popeye" deformity as the primary outcome variable of interest.

We take into account statistics reported in literature, which indicate a "Popeye sign" frequency of $41-43 \%$ after tenotomy, and $8-25 \%$ after tenodesis ${ }^{11,12}$. If we consider the least difference between these techniques $(16 \%=41-8 \%)$, than the study should be conducted in groups composed of more than 134 subjects, otherwise considering the maximum difference $(37 \%=43-25 \%)$, it should be conducted in groups composed of more than 23 subjects.

If the difference found in the test is particularly high, it is still possible to consider the number of patients statistically significant: by presuming a $50 \%$ difference between the two techniques while keeping all other indicators unchanged (power $80 \%$ and significance $5 \%$ ), a sample of 12 subjects for each group would be sufficient.

The Fisher's exact test is used to verify if the presence of a "Popeye sign" is significantly inferior after tenodesis than following tenotomy. Since the sample is not particularly wide and the frequency with which the symptoms occur is low, we choose this test over the 2 test.

\section{Surgical technique}

Patient in the beach-chair position. A 3-cm cutaneous incision is made in the projection of the proximal part of the Pectoralis Major, adjacent to the deltopectoral interval (Fig. 1). Through this space the Pectoralis

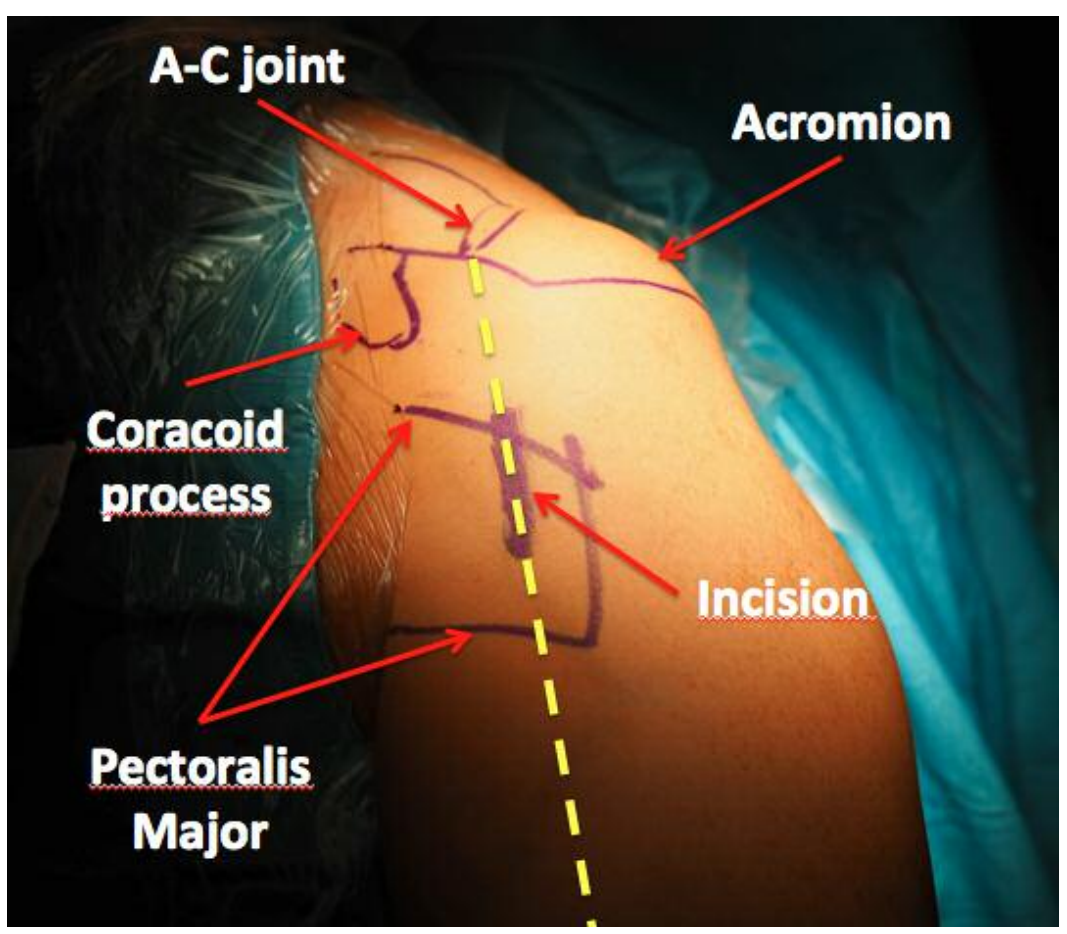

Figure 1. Patient in the beach chair position. Anterior view of the left shoulder. The incision line is made along an imaginary line (yellow line) that originates from the acromioclavicular joint (A-C joint) and ends at the distal humeral biceps tendon. It has to be made straddling the cranial margins of the pectoralis major tendon. 


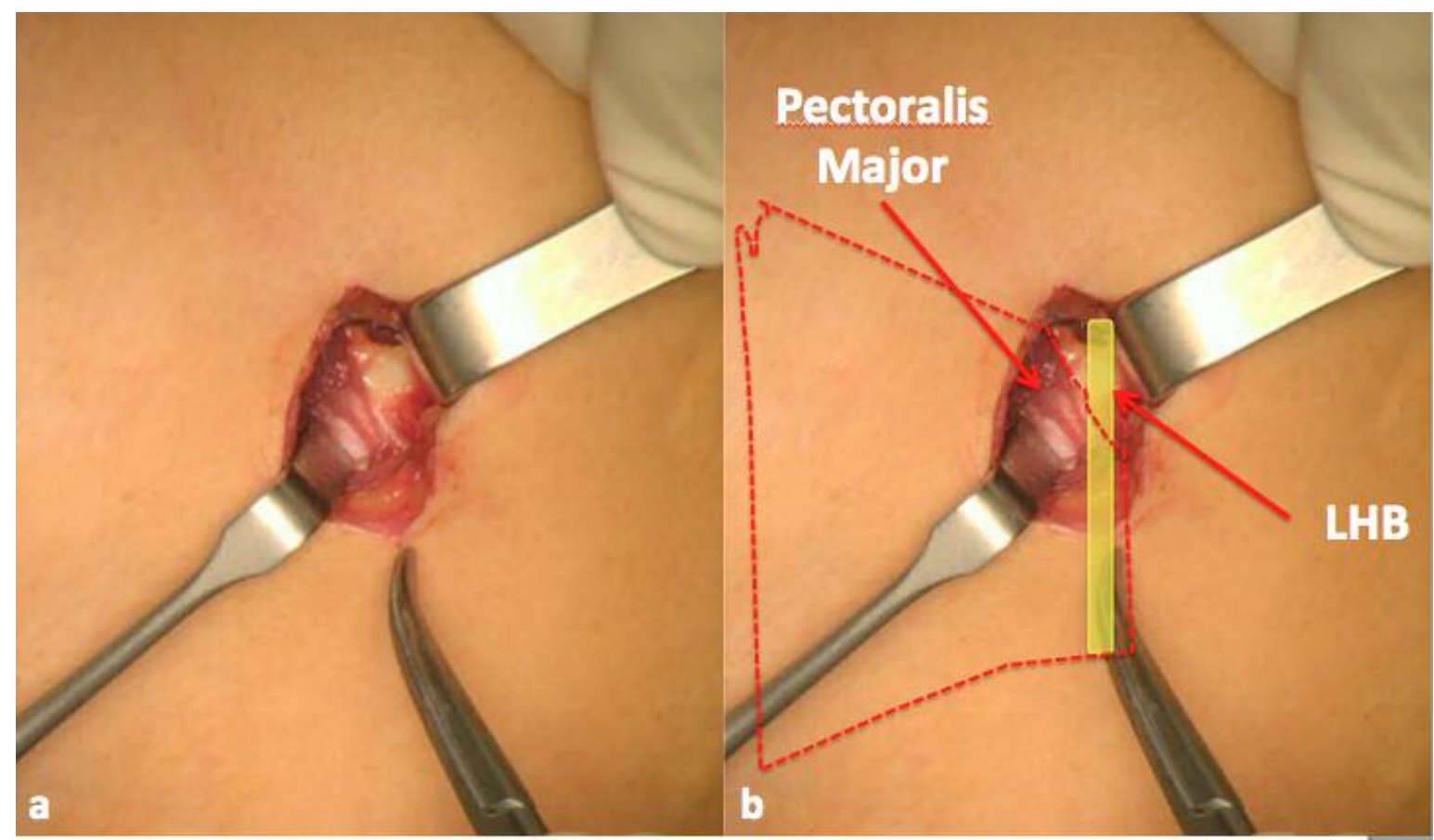

Figure $2 \mathrm{a}, \mathrm{b}$. a) Through the deltopectoral space, we reach the pectoralis major tendon proximal margin (dotted line in red Fig. b); medially to its insertion the long head of biceps can be palpated (yellow rectangle Fig. b).

Major is reached then incised just medially to its humeral insertion, in line with the fibres direction, thus exposing the LHB (Fig. 2). The LHB tenodesis to the Pectoralis Major tendon (Fig. 3) is performed by two resorbable sutures (Vycril Nr. 2, Ethicon Inc. Somerville, NJ). The skin is then closed and we move to the arthroscopy. The LHB tenotomy follows the joint inspection. The biceps stump is then removed from the bicipital groove to rule out that a pathology of this extra-articular tract could cause residual pain. Next, we proceed with the other surgical steps according to existing pathologies.

\section{Postoperative management}

Postoperative management was the same for both groups, according to concurrent procedures: in case of a rotator cuff tear, we prescribed a sling in abduction for 5 weeks; in the other cases, we prescribed a sling just for the patient's comfort. During the post-op period, elbow movements were limited for three weeks, and loads forbidden for at least 2 months.

\section{Results}

According to the exclusion/inclusion criteria, we selected 22 consecutive patients treated with tenodesis, and 27 patients treated with tenotomy. Among them, $16(70 \%)$ and $16(59 \%)$ subjects in each group were available for follow-up. Of the remaining 17 patients, 5 were not traceable, 10 were not available for followup due to distance, 2 recently underwent surgery not related to the shoulder. The mean age at the evalua-

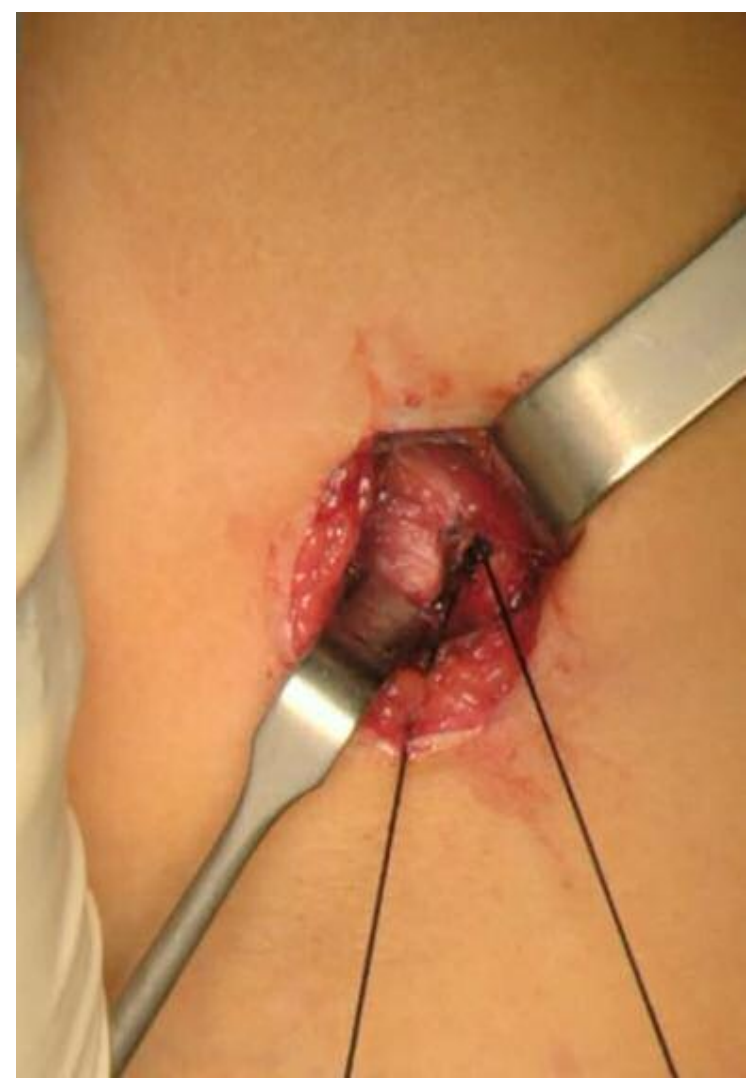

Figure 3. The long head biceps is sutured to the pectoralis major tendon by Vycril Nr. 2 (Ethicon Inc. Somerville, NJ) resorbable suture. 
Table I. Associated lesions and concomitant procedures. Long head biceps pathology only, without concomitant lesions.

\begin{tabular}{llll}
\hline Diagnosis & Concomitant procedures & Tenodesis & Tenotomy \\
\hline LHB & None & 3 & 1 \\
Rotator cuff lesion & Cuff repair & 13 & 10 \\
Capsulitis & Arthroscopic capsular release & 0 & 5 \\
\hline
\end{tabular}

Table II. Subjective symptoms concern patient's perception: if he/she feels anterior pain during movements, if he/she experienced spasms in the biceps brachii muscle belly during follow-up or post-op periods, if he/she perceives a visual or strength difference between the biceps. The clinical signs are obtained by the tests administered during follow-up, as the speed's test or the Yergason test, painfulness in the humeral groove region, and the presence or absence of the "Popeye sign". The scores are the mean points obtained by the two groups on the administered scoring systems.

\begin{tabular}{|c|c|c|}
\hline & Tenodesis & Tenotomy \\
\hline \multicolumn{3}{|c|}{ Subjective symptoms } \\
\hline Anterior pain & 1 & 2 \\
\hline Muscular spasm at f.u. & 1 & 5 \\
\hline Muscular spasm after surgery & 1 & 5 \\
\hline Aesthetic difference & 1 & 4 \\
\hline Strength difference & 2 & 3 \\
\hline \multicolumn{3}{|c|}{ Clinical signs } \\
\hline Popeye, mild & 1 & 3 \\
\hline Popeye, severe & 0 & 6 \\
\hline Bicipital groove pain & 0 & 5 \\
\hline Speed test & 1 & 3 \\
\hline Yergason test & 0 & 0 \\
\hline \multicolumn{3}{|c|}{ Scores } \\
\hline ASES (mean) & 92.9 & 90.8 \\
\hline SPADI (mean) & 92.5 & 89.7 \\
\hline SST (mean) & 8.9 & 8.4 \\
\hline
\end{tabular}

tion was 54 years (8 SD; 43-75) for tenodesis, and 56 years (9 SD; 45-75) for tenotomies. The mean followup was 14.7 months: 13.5 months (2.12 SD; 10-17) for tenodesis, 15.8 (4.54 SD; 11-21) for tenotomies. Associated lesions and concomitant procedures are listed in Table I.

The mean ASES Score was 92.9 (11.2 SD; 55-100) for tenodesis, and 90.8 (13.38 SD; 50-100) for tenotomy. The mean SPADI score was 92.5 (10.26 SD; 61 100) for tenodesis, and 89.7 (10.62 SD; 67-100) for tenotomy. The mean SST score was 8.9 (1.28 SD; 5.8-10) for tenodesis and 8.4 (1.69 SD; 5-10) for tenotomy. Data do not follow a normal distribution, as established by the Saphiro-Wilk test of normality, therefore it is not possible to compare the average scores since they are not a valid evaluation source of the data distribution itself. With the Wilcoxon signedrank test and a 0.05 confidence level, there is no statistically significant difference between the two groups for all three scores.

The results of the clinical evaluation are summarised in Table II.
The "Popeye sign" was significantly more frequent in the TT group (56.25\%) than in the TD group $(6.25 \%)$. When present, we classified it as mild (minimal bunch of the muscle belly visible in its proximal portion) or severe (striking bunch of the muscle belly). In this case too, the TT group showed worse results: 3 mild, and 6 severe "Popeye sign", versus 1 mild "Popeye sign" in the TD group. The Fisher's exact test shows a $p$-value of 0.002961 . By using a significance level of 0.05 , the $p$-value is lower, and it is thereby possible to reject the null hypothesis that asserts the equality between the two samples. De facto, we can allow that the tenodesis technique shows a significant (for a significance level $a=0.05$ ) inferior presence of "Popeye sign" compared to the tenotomy technique. Also according to the other analysed parameters, the TT group showed a major percentage of positive signs/symptoms (Tab. II). The strength difference perceived by the patient compared to the contralateral arm tested similarly in the two groups (difference perceived in 2 tenodesis cases, and in 3 tenotomy cases). 
Patients who showed a positive "Popeye sign" had a major percentage of symptoms $(61 \%)$, regardless of the group to which they belonged. By using the Fisher's exact test we prove that patients showing a "Popeye sign" are more likely (for a significance level $a=$ 0.05 ) to report other symptoms ( $p$-value $=0.014931$ ). In fact, by using a significance level of 0.05 , the $p$ value is inferior, thus allowing the rejection of the null hypothesis that asserts the lack of predisposition among subjects showing a "Popeye sign" to develop other symptoms.

The most frequently occurred symptom in the TD group was the patient perception of a strength difference between the two biceps (in the $12.5 \%$ of the cases). In the TT group, the more frequent symptoms, that occurred in the $31.25 \%$ of the patients, were the presence of spasms in the humeral biceps muscle belly (both during follow-up and post-op periods) and soreness during pressure in the humeral groove.

No patient showed signs of infection, nerve or vascular lesions.

\section{Discussion}

The principal findings of this study show that this new tenodesis technique of the long head of the biceps proved to obtain better results than simple tenotomy in terms of subjective and objective symptomatology. Symptoms shown by patients who underwent tenotomy, and absent in those who underwent tenodesis, are still not debilitating and do not affect the scores results.

Tenotomy is definitively a simpler and more reproducible technique than tenodesis but it is likely to cause a "popeye deformity" in $3-70 \%$ of cases; it is not recommended in a young and active population, since in $38 \%$ of cases can result in fatigue cramping of the biceps muscle belly ${ }^{13}$.

LHB suture to the soft tissues (soft tissue tenodesis), a largely used technique and can be easily performed arthroscopically; the LHB can be sutured to the rotator cuff ${ }^{14}$, to the rotator interval ${ }^{15}$ or to the conjoint tendon ${ }^{16}$. The tendon fixation to the bone provides a greater gliding resistance but does not mimic the biomechanical characteristics of the native tendon; on the contrary, as proved by a cadaveric study, it produces a significantly greater strain than the soft-tissue tenodesis ${ }^{17}$.

There is no consensus regarding the best location to perform the tenodesis. Some Authors believe that the subpectoral fixation is the best option, since by removing the proximal portion of the tendon from the biceps groove, the risk of a residual "groove pain" would decrease ${ }^{3}$. Both Lutton et al. ${ }^{18}$ and Sanders et al. ${ }^{19}$ registered a significant increase of complications and recurrent pain in the fixations proximal to the pectoralis major. Brady et al. ${ }^{20}$ later downsized this risk by examining a wide series of patients (1083 cases), recording the $0.4 \%$ of complications with an into the groove fixation using interference screws.
The subpectoral fixation requires to drill the bone; the risk of fracture, even rare, is tangible. The fracture usually occurs during the first months of the post-op period. The adverse event is due to the diameter, depth and position of the hole drilled to accommodate the fixation device. This hole increases the mechanical stress on the humeral cortex. This complication is currently reported in literature by several Authors through case reports ${ }^{7}, 21-24$. Drilling the posterior cortex exposes patients to the risk of nerve lesions. The described cases include musculocutaneous nerve transient palsy ${ }^{25}$, musculocutaneous nerve entrapment $^{26}$, and severe neurological lesions upon the median, radial, ulnar, and musculocutaneous nerves ${ }^{6}$.

The LHB tenodesis performed by the proximal tendon excision as first step can miss the proper tension to the muscle belly. Cadaveric studies proved that open subpectoral tenodesis allows a better repositioning of the tendon compared to arthroscopic suprapectoral tenodesis, still resulting in an overtensioning of the muscle belly 27 .

For these reasons, we developed a tenodesis technique that can be performed with the proximal portion of the tendon still intact, thus in the right tension, that avoids trans-bone holes and allows to remove the tendon from the groove by removing its proximal stump as final step. This technique is reproducible, performed through a small $2-3 \mathrm{~cm}$ skin incision matching the pectoralis major tendon at the deltopectoral interval, whose window is closed by sutures at the end of the tenodesis. The following arthroscopy is not influenced by this procedure, neither by fluid leakage. Thanks to this technique, the fixation resistance is less rigid compared to the one obtained through a trans-bone method and respects the physiological tensions of the tendon in vivo; however, as shown by the results, it is able to guarantee its anchoring.

With a mean follow-up of 14.7 months, this study proves that the "no-holes trans-pectoral" technique is reliable and repeatable.

The absence of a significant difference between the scores in favour of tenotomy is because the issue of postoperative pain, that leds patients from two respectively homogenous groups to surgery, is properly addressed by both techniques. The tenotomy residual complications marginally affect the clinical and functional results, therefore the scores.

The frequency of "popeye deformity" among the TT and the TD groups is similar to those found in the literature: $41-43 \%$ in tenotomies, and $8-25 \%$ in tenode$\mathrm{sis}^{25-27}$. Moreover, it has been shown that tenotomy increases patient's symptoms (cramping), even without outlining a statistically significant difference ${ }^{28}$. In our study, we found a higher statistically significant frequency of "Popeye" deformity in the TT group. Moreover, we recorded a higher frequency of subjective symptoms in patients treated by tenotomy, reported as mild discomfort rather that debilitating symptomologies.

This new technique obtains similar outcome to other procedures reported in the literature and provides several benefits. The original biomechanical benefits 
include the maintenance of the physiological tension of the LHB, still inserted at both two ends during the tenodesis, along with the fixation at its own course. The economic benefit is that the cost is limited to the resorbable sutures (Vycril 2); moreover, potentially, it does not cause the same complications described about other techniques, the trans-bone ones specially. The limitations of the study are due to its retrospective design and to the short follow-up period. Moreover, it is rare that a patient undergoes surgery solely and exclusively for LHB pathologies; the study group, therefore, reaches a statistically significant number of subjects only by including patients with other associated pathologies, as small cuff lesions and adhesive capsulitis, even if the inclusion criteria are very strict.

\section{Conflicts of interest}

The Authors declare no conflicts of interest concerning this article.

\section{References}

1. Patel KV, Bravman J, Vidal A, Chrisman A, McCarty E. Biceps tenotomy versus tenodesis. Clin Sports Med. 2016;35:93-111.

2. Biz C, Vinanti GB, Rossato A, Arnaldi E, Aldegheri R. Prospective study of three surgical procedures for long head biceps tendinopathy associated with rotator cuff tears. Muscle Ligaments Tendons J. 2012;2(2):133-136.

3. Virk MS, Nicholson GP. Complications of proximal biceps tenotomy and tenodesis. Clin Sports Med. 2016;35:181-188.

4. Alpantaki K, McLaughlin D, Karagogeos D, Hadjipavlou A, Kontakis G. Sympathetic and sensory neural elements in the tendon of the long head of the biceps. J Bone Joint Surg Am. 2005;87:1580-1583.

5. Voss A, Cerciello S, Yang J, Beitzel K, Cote MP, Mazzocca AD. Open subpectoral tenodesis of the proximal biceps. Clin Sports Med. 2016;35:137-152.

6. Rhee PC, Spinner RJ, Bishop AT. latrogenic brachial plexus injuries associated with open subpectoral biceps tenodesis: a report of 4 cases. Am J Sports Med. 2013;41(9):2048-2053.

7. Sears BW, Spencer EE, Getz CL. Humeral fracture following subpectoral biceps tenodesis in 2 active, healty patients. $J$ Shoulder Elbow Surg. 2011;20(6):e7-11.

8. Padua R, Padua L, Ceccarelli E, Bondi R, Alviti F, Castagna A. Italian versione of ASES questionnaire for shoulder assessment: cross-cultural adaptation and validation. Musculoskelet Surg. 2010;94:S85-S90.

9. Marchese C, Cristalli G, Pichi B, Manciocco V, Mercante G, Pellini R, et al. Italian cross-cultural adaptation and validation of three different scales for the evaluation of shoulder pain and dysfuction after neck dissect: University of California - Los Angeles (UCLA) Shoulder Scale, Shoulder Pain and Disability Index (SPADI) and Simple Shoulder Test (SST). Acta Otorhinolaryngol Ital. 2012;32:12-17.

10. Padulo J, Oliva F, Frizziero A, Maffulli N. Muscles, Ligaments and Tendons Journal - Basic principles and recommendations in clinical and field science research: 2016 update. MLTJ. 2016;6(1):1-5.
11. Werner BC, Lyons ML, Evans CL, Griffin JW, Hart JM, Miller MD, Brockmeier SF. Arhroscopic suprapectoral and open subpectoral biceps tenodesis: a comparison of restoration of length.tension and mechanical strength between techniques. Arthroscopy. 2015;31(4):620-627.

12. Hsu AR, Ghodadra NS, Provencher MT, Lewis PB, Bach BR. Biceps tenotomy versus tenodesis: a review of clinical outcomes and biomechanical results. J shoulder Elbow Surg. 2011;20:326-332.

13. Nho SJ, Strauss EJ, Lenart BA, Provencher MT, Mazzocca $A D$, Verma NN, Romeo AA. Long head of the biceps tendinopathy: diagnosis and management. J Am Acad Orthop Surg. 2010;18:645-656.

14. Franceschi F, Longo UG, Ruzzini L, Papalia R, Rizzello G, Denaro V. To detach the long head of the biceps tendon after tenodesis or not: outcome analysis ot the 4 year follow-up of two different techniques. International Orthopaedics. 2007; 31:537-545.

15. Elkousy HA, Fluhme DJ, O'Connor DP, Rodosky MW. Arthroscopic biceps tenodesis using the percutaneous, intra-articular trans-tendon technique: preliminary results. Orthopedics. 2005;28:1316-1319.

16. Verma N, Drakos M, O'Brien S. Arthroscopic transfer of the long head of the biceps to the conjoint tendon. Arthroscopy. 2005;21(6):764.

17. Levin SD, Wellman DS, Liu C, Li Y, Ren Y, Shah NA, Zhang LQ. Biomechanical strain characteristics of soft tissue biceps tenodesis and boy tenodesis. J Orthop Sci. 2013;18:699-704.

18. Lutton DM, Gruson KI, Harrison AK. Where to tenodese the biceps: proximal or distal? Clin Orthop Relat Res. 2011;469: 1050-1055.

19. Sanders B, Lavery KP, Pennington S. Clinical success of biceps tenodesis with and without release of the transverse humeral ligament. J Shoulder Elbow Surg. 2012;21:66-71.

20. Brady PC, Narbona P, Adams CR, et al. Arthroscopic proximal biceps tenodesis at the articular margin: evaluation of outcomes, complications and revision rate. Arthroscopy. 2015;31:470-476.

21. Friedel R, Markgraf E, Schmidt I, Donicke T. Proximal humerus shaft fracture as a complicazion after keyhole-plasty. A case report. Unfallchirurgie. 1995;21:198-201.

22. Gyulai M. Humeral fracture after keyole tenodesis. MagyTraumatol Orthop Helyreallito Seb. 1990;33:234-236.

23. Reiff SN, Nho SJ, Romeo AA. Proximal humerus fracture after keyhole biceps tenodesis. Am J Orthop (Belle Mead NJ). 2010;39:e61-3.

24. Dein EJ, Huri G, Gordon JC, McFarland EG. A humerus fracture in a baseball pitcher after tenodesis. Am J Sports Med. 2014:42:877-879.

25. Nho SJ, Reiff SN, Verma NN, Slabaugh MA, Mazzocca AD, Romeo AA. Complications associated with subpectoral biceps tenodesis: low rates of incidence following surgery. J Shoulder Elbow Surg. 2010;19(5):764-768.

26. Ma H, Van Heest A, Glisson C, Patel S. Musculocutaneous nerve entrapment: an usual complication after biceps tenodesis. Am J Sports Med. 2009;37(12):2467-2469.

27. Slenker NR, Lawson K, Ciccotti MG, Dodson CC, Cohen SB. Biceps tenotomy versus tenodesis: clinical outcomes. Arthroscopy. 2012;28:576-582.

28. Leroux T, Chahal J, Wasserstein D, Verma NN, Romeo AA. A systematic review and meta-analysis comparing clinical outcomes after concurrent rotator cuff repair and long head biceps tenodesis or tenotomy. Sports Health. 2015;7(4):303307. 